\title{
Overt lymph node metastases from a gastrointestinal stromal tumor of the esophagus
}

\author{
Takaaki Masuda, MD, PhD, ${ }^{a}$ Yasushi Toh, MD, PhD, ${ }^{a}$ Akira Kabashima, MD, PhD, ${ }^{a}$ Yoshiro Aoki, MD, ${ }^{a}$ \\ Norifumi Harimoto, MD, PhD, ${ }^{a}$ Shuhei Ito, MD, PhD, ${ }^{a}$ Junya Taomoto, MD, PhD, ${ }^{a}$ Osamu Ikeda, MD, PhD, ${ }^{a}$ \\ Takefumi Ohga, MD, PhD, ${ }^{a}$ Eisuke Adachi, MD, PhD, ${ }^{a}$ Yoshihisa Sakaguchi, MD, PhD, ${ }^{a}$ Minako Hirahashi, MD, PhD, \\ Kenichi Nishiyama, MD, PhD, and Takeshi Okamura, MD, PhD, ${ }^{\mathrm{a}}$ Fukuoka, Japan
}

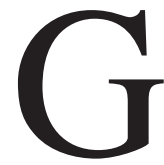
astrointestinal stromal tumor (GIST) is the most common mesenchymal neoplasm with malignant potential that arises from the c-KIT (CD117)-positive intestinal cells of Cajal in the gastrointestinal tract. ${ }^{1}$ GIST accounts for $0.1 \%$ to $3.0 \%$ of all tumors in the gastrointestinal tract and occurs in $60 \%$ of tumors in the stomach, $35 \%$ of tumors in the small bowel, and less than $1 \%$ of tumors in the esophagus. ${ }^{1}$ Metastases of GIST commonly develop in the abdominal cavity and liver, and rarely in the lymph nodes (LNs). ${ }^{1,2}$ The standard treatment of GIST is a complete surgical resection without a regional lymphadenectomy. ${ }^{3}$ We describe a rare case of LN metastases from an esophageal GIST.

\section{Clinical Summary}

A 52-year-old man was hospitalized with a 1-month history of chest pain and dysphagia. His medical and family history were unremarkable. He had a 30-year history of smoking 25 cigarettes per day and occasionally drank alcohol. An esophagogastroscopy showed an ulcerated large mass partially covered with normal mucosa, which was located on the left wall of the esophagus $34 \mathrm{~cm}$ from the incisors. Another lesion with an ulcerated elevation, which was almost completely covered with normal mucosa, was found at the gastric fundus. A barium swallow showed a welloutlined tumor of $8 \times 4 \mathrm{~cm}$ on the left esophageal wall of the lower third of the thoracic esophagus and an elevated lesion with an ulcer at the gastric fundus (Figure 1, A). A computed tomographic scan revealed thickening of the wall extending from the distal esophagus to the gastric fundus and a well-outlined tumor in the left lateral esophageal wall involving the lumen of the gastric fundus (Figure 1,B). An apparent lymphadenopathy was observed around the distal esophagus and gastric fundus (Figure 1, B). Distant metastases, such as in the liver and lung, were not observed. The tumor was preoperatively diagnosed to be an esophageal,

\footnotetext{
From the Departments of Gastroenterogical Surgery ${ }^{\mathrm{a}}$ and Pathology, National Kyushu Cancer Center, Fukuoka, Japan.

Received for publication May 23, 2007; accepted for publication June 1, 2007.

Address for reprints: Yasushi Toh, MD, PhD, Department of Gastroenterogical Surgery, National Kyushu Cancer Center, 3-1-1, Notame, Minami-ku, Fukuoka 811-1395, Japan (E-mail: ytoh@nk-cc.go.jp).

J Thorac Cardiovasc Surg 2007;134:810-1

$0022-5223 / \$ 32.00$

Copyright $\odot 2007$ by The American Association for Thoracic Surgery doi:10.1016/j.jtcvs.2007.06.002
}

poorly differentiated squamous cell carcinoma with regional LN metastases according to the clinical and histopathologic findings.

A subtotal esophagectomy reconstructed with a gastric tube through the retrosternal route was performed to completely resect the tumor with dissection of cervical, mediastinal, and abdominal LNs. The patient had an uneventful postoperative recovery.

The tumor was a well-circumscribed, gray-white fibrous mass measuring $9.5 \mathrm{~cm}$, involving the entire layer of the esophagus (Figure 2, A). A histopathologic examination showed the interlacing bundles or whorls of spindle-shaped cells with elongated blunt-ended nuclei (Figure 2, A). The mitotic index was 15/50 in high-power fields. LN metastases around the gastric fundus were histologically confirmed (Figure 2, B). Immunohistochemical studies showed the tumor cells to stain diffusely for c-KIT and CD34, and not for S-100 or $\alpha$-smooth muscle actin.

This tumor was diagnosed to be esophageal GIST with highgrade malignancy based on the International Consensus on GIST criteria. $^{3}$

The patient is asymptomatic without any evidence of recurrence 18 months after surgery with the adjuvant administration of imatinib (Gleevec, Novartis, Basel, Switzerland), which is a signaltransduction inhibitor of c-KIT, for 12 months as a clinical trial.

\section{Discussion}

Most GISTs exhibit activating mutations of c-KIT and serve as a model for molecular-based diagnosis and treatment. ${ }^{1}$ The preoperative diagnosis of GIST is difficult in any region mainly because of the low frequency and difficulty of the biopsy.

In this case, the ulcerated tumor was located in the distal esophagus and had regional LN metastases. The clinical findings of most cases suggest the presence of an epithelial malignant tumor and led to the false preoperative diagnosis of an undifferentiated esophageal cancer that was morphologically similar to GIST. To our knowledge, this is the first case report of overt LN metastases from an esophageal GIST.

Miettinen and colleagues ${ }^{4}$ reported that 48 of 68 esophageal mesenchymal tumors $(71 \%)$ were leiomyomas and 17 (25\%) were GISTs, and that 12 of 17 esophageal GISTs (71\%) were classified as high-grade malignancies. This study indicates that GISTs are rare among esophageal mesenchymal tumors, although many of them are classified as high-grade malignancies. The present case is asymptomatic without recurrence at 18 months after surgery despite the high-grade malignancy with LN metastases. This may be due to the complete surgical resection with extensive dissection of LNs and the adjuvant administration of imatinib., 


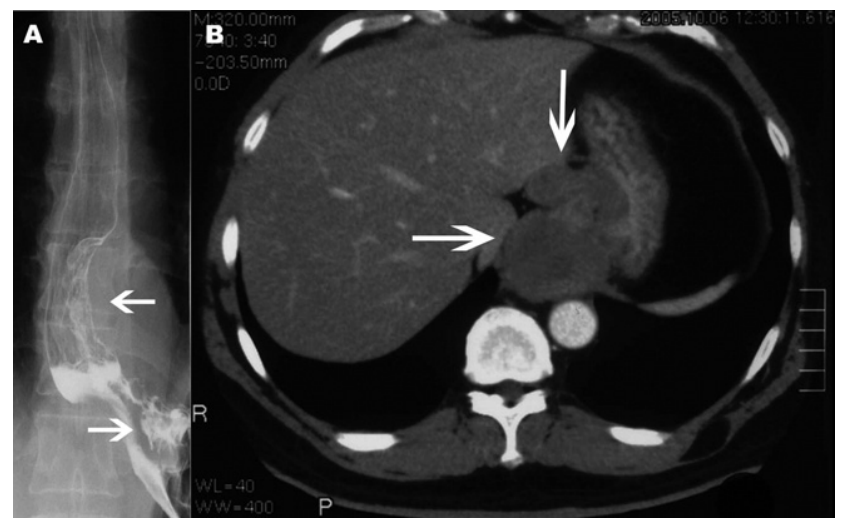

Figure 1. A, Barium swallow showed a well-outlined tumor at the left esophageal wall of the lower third of the thoracic esophagus and an elevated lesion with an ulcer at the gastric fundus. B, Computed tomographic scan revealed thickening of the esophageal wall and lymphadenopathy around the distal esophagus and gastric fundus.

This seems to be a rare case of esophageal GIST with LN metastases. Diagnosis of an esophageal tumor should thus include the possibility of GIST, notwithstanding the presence of LN metastases. Immunohistochemistry with c-KIT is a key tool for this diagnosis. Analyses of the clinical, histopathologic, and molecular-biologic characteristics of GIST with LN metastases would help in selecting the optimal treatments for GIST, while shedding light on the mechanism of the malignant progression of GIST.

We thank Dr Hidetaka Masuda for critical advice.

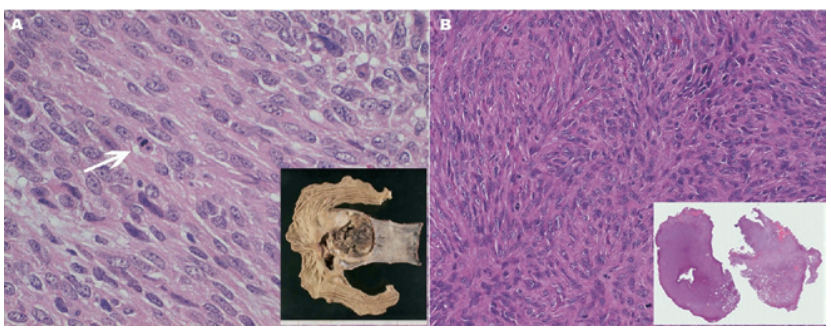

Figure 2. A, The resected tumor was a well-circumscribed, graywhite fibrous mass. The tumor was composed of interlacing bundles or whorls of spindle-shaped cells with elongated bluntended nuclei. Mitotic figures were recognized (hematoxylineosin). B, LN metastases around the gastric fundus were histologically confirmed (hematoxylin-eosin).

\section{References}

1. Miettinen M, Lasota J. Gastrointestinal stromal tumors: review on morphology, molecular pathology, prognosis, and differential diagnosis. Arch Pathol Lab Med. 2006;130:1466-78.

2. Fong Y, Coit DG, Woodruff JM, Brennan MF. Lymph node metastasis from soft tissue sarcoma in adults. Analysis of data from a prospective database of 1772 sarcoma patients. Ann Surg. 1993;217:72-7.

3. Blay JY, Bonvalot S, Casali P, Choi H, Debiec-Richter M, Dei Tos AP, et al. Consensus meeting for the management of gastrointestinal stromal tumors. Report of the GIST Consensus Conference of 20-21 March 2004, under the auspices of ESMO. Ann Oncol. 2005;16:566-78.

4. Miettinen M, Sarlomo-Rikala M, Sobin LH, Lasota J. Esophageal stromal tumors: a clinicopathologic, immunohistochemical, and molecular genetic study of 17 cases and comparison with esophageal leiomyomas and leiomyosarcomas. Am J Surg Pathol. 2000;24:211-22.

5. Bumming P, Andersson J, Meis-Kindblom JM, Klingenstierna H, Engstrom K, Stierner U, et al. Neoadjuvant, adjuvant and palliative treatment of gastrointestinal stromal tumours (GIST) with imatinib: a centrebased study of 17 patients. Br J Cancer. 2003;89:460-4. 